\title{
Robotic Surgery in the Obese Patient: Tips and Tricks for the Benign Gynecologist
}

\section{Hana Mikdachi ${ }^{*}$ and Arielle Schreck ${ }^{2}$}

${ }^{1}$ Division of Minimally Invasive Gynecologic Surgery, Department of Obstetrics and Gynecology, East Tennessee State University, USA ${ }^{2}$ Department of Obstetrics and Gynecology, East Tennessee State University, Johnson City, Tennessee, USA

\section{Abstract}

\section{Publication History:}

Robotic-assisted surgery in gynecologic procedures continues to increase in numbers with several Received: November 23,2018 advantages over other types of minimally invasive surgery. Obese patients undergoing robotic surgery have Accepted: December 20, 2018 shorter hospital stays, less blood loss, lower conversion rates and lower postoperative complications despite Published: December 22,2018 the increase in surgical complexity of their cases.

Obese patients have the highest need for minimally invasive surgery because they have increased perioperative morbidity and mortality rates, as well as worse surgical outcomes and complications with increasing BMI. Minimally invasive surgery reduces the risk of venous thromboembolism, wound infections, ileus and postoperative fevers in obese women.

\section{Keywords:}

Benign gynecology, Obesity, Obese patients, Robotic surgery

Robotic-assisted surgery offers a minimally invasive surgical approach to the obese woman who cannot have vaginal or conventional laparoscopic surgeries due to the physical limitations of her redundant vaginal sidewall tissue and thick abdominal wall. The robotic approach takes less operating time than conventional laparoscopic surgery in the super morbidly obese population, and surgeons experience less fatigue and mental stress.

In this review article, we provide the benign gynecologist with recommendations for the preoperative and postoperative periods when performing robotic-assisted surgery on the obese gynecologic patient. We also offer detailed suggestions for effective patient positioning of even the most super morbidly obese patients. We also explain several techniques to enter the abdomen, the step which often challenges the surgeon the most and can lead to pre-peritoneal insufflation and sub-optimal visualization during the case.

\section{Introduction}

The use of robotic surgery in gynecologic procedures continues to increase. Annually, a nationwide sample in the United States reported that the percentage of hysterectomies performed robotically increased from $0.9 \%$ in 2008 to $8.2 \%$ in 2010 [1].

Various reasons contributed to this increase in popularity of robotic surgery. A review of hysterectomy cases between 2007 and 2010 at two large health system teaching hospitals demonstrates that robotic surgery is associated a shorter length of hospital stay and less blood loss $(50 \mathrm{~mL})$ compared to other minimally invasive hysterectomy $(\mathrm{MIH})$ types in which average blood loss ranges from $150 \mathrm{~mL}$ (traditional laparoscopy) to $250 \mathrm{~mL}$ (vaginal approach) [2]. Therefore, a robotic approach may benefit patients in whom significant blood loss is anticipated at time of hysterectomy, and in those who cannot afford to lose blood such as the anemic patient.

New data from a Swedish population study of 13,806 hysterectomies performed for benign indications between 2009 and 2015 report lower conversion rates for robotic-assisted hysterectomies (RAH) than laparoscopic hysterectomies (LH) and vaginal hysterectomies (VH); the reported conversion rates are $10 \%$ for $\mathrm{LH}, 4.8 \%$ for $\mathrm{VH}$ and $1.6 \%$ for RAH [3]. The same study provides evidence of shorter operating times for RAH (104 min) than LH (127 min) despite longer operating times than vaginal hysterectomies $(\mathrm{VH})$ and abdominal hysterectomies (AH), reported as $75 \mathrm{~min}$ and $97 \mathrm{~min}$ respectively [3].

Because of this increase in operating time without major differences in primary outcomes, some authors advocate for the $\mathrm{VH}$ as the hysterectomy of choice whenever feasible, a statement endorsed by the American College of Obstetricians and Gynecologists (ACOG) [4]. However, a Cochrane Review of 47 randomized controlled trials (RCT) of hysterectomy for benign gynecologic disease involving 5,102 women reports no difference in MIH types in the primary outcomes of return to normal activities, satisfaction, quality of life, intraoperative visceral injury and major long-term complications [5].

Data from high-volume surgeons with more robotic experience refute these claims in a large multicenter analysis comparing data from 2,300 RAH, 9,745 $\mathrm{AH}, 8,121 \mathrm{VH}$ and 11,952 $\mathrm{LH}$ with evidence of lower postoperative complication rates in the RAH group despite increased complexity with higher rates of adhesive disease, large uteri and morbid obesity [6]. These numbers and studies provide convincing information that RAH offers distinct advantages over $\mathrm{LH}$, and as a platform, robotic surgery stands to stay and become the new normal.

The substantial obesity rates in the United States and worldwide opens a wide lane for robotic surgery by offering a minimally invasive

"Corresponding Author: Dr. Hana Mikdachi, Division of Minimally Invasive Gynecologic Surgery, Department of Obstetrics and Gynecology, East Tennessee State University, Johnson City, Tennessee, USA, Tel: 423-439-7272; Fax: 423-4397235; E-mail: eladomikdach@mail.etsu.edu

Citation: Mikdachi H, SchreckA (2018) Robotic Surgery in the Obese Patient: Tips and Tricks for the Benign Gynecologist. Int J Gynecol Clin Pract 5: 146. https://doi. org/10.15344/2394-4986/2018/146

Copyright: (c) 2018 Mikdachi et al. This is an open-access article distributed under the terms of the Creative Commons Attribution License, which permits unrestricted use, distribution, and reproduction in any medium, provided the original author and source are credited. 
surgical approach to patients who might otherwise not be candidates. Data from the Centers for Disease Control and Prevention National Health and Nutrition Examination Survey report the prevalence of obesity from 2015 to 2016 as $41.5 \%$ for women [7]. The World Health Organization reports that worldwide obesity has tripled since 1975 [8]. In 2016, more than 1.9 billion adults aged 18 years and older were overweight and 650 million were obese, which equates to $40 \%$ and $15 \%$ of women, respectively [8]. With the concurrent increases in popularity of robotic surgery and obesity rates, the benign gynecologist may want to sharpen her or his robotic skills in the obese patient.

\section{Rationale for Robotic Surgery in the Obese Patient}

\section{Importance of minimally invasive surgery(MIS) in the obese patient}

Obesity as a risk factor correlates with higher perioperative morbidity and mortality rates, and worse surgical outcomes such as increased risk of myocardial infarction, peripheral nerve injury, surgical site infections (SSI) and urinary tract infections [9]. MIS may help to mitigate the rates of these complications.

Patients who undergo MIS ambulate more quickly which may reduce the risk of venous thromboembolism (VTE). An analysis of 1,112 women undergoing surgery for endometrial carcinoma or hyperplasia between 2008 and 2014 report higher rates of VTE as body mass index (BMI) increases with rates of $0.2 \%$ for a BMI of 29 or less and $3 \%$ for a BMI greater than 40 [10]. The study only demonstrates this increase in patients who had a laparotomy with no difference in patients who underwent MIS [10].

The same authors also note an increase in wound infection from $3 \%$ to $7 \%$ in non-obese and obese cohorts with the same conclusion that obesity only increases this risk in patients undergoing laparotomy [10]. Other studies of gynecologic surgery in obese patients comparing $\mathrm{AH}$ to MIH also report higher rates of wound dehiscence and wound infection in the open surgery group $[11,12]$.

Surgeons who perform $\mathrm{MIH}$ as an alternative to $\mathrm{AH}$ in obese women reduce the length of their patients' hospital stays by several days [11-13]. The shorter length of hospital stays translates to increased hospital cost savings. Women who undergo MIH reduce hospital costs compared to those who have a laparotomy, and morbidly obese women have the highest cost difference [10]. MIS offers a higher value of care by decreasing complications and costs, especially in morbidly obese women.

In addition to lowering risks of VTE and SSIs, decreased length of hospital stay and reducing costs, MIS correlates with less ileus and postoperative fevers in obese women in studies of both simple and complex pelvic surgery [14-16]. ACOG emphasizes the value of MIS in reducing poor outcomes and acknowledges the challenges with visualization during vaginal surgery in obese women, such as the size of the thighs and buttocks and redundant vaginal sidewalls [17]. Many gynecologic surgeons may prefer laparoscopic and robotic approaches when operating on obese women.

\section{Importance of robotic surgery in the obese patient}

Robotic surgery offers distinct advantages compared to other minimally invasive approaches in the obese patient. A substantial portion of the research on robotics in the obese and especially the morbidlyobesepopulation stems fromitssuccessin the arena ofbariatric surgery, where bariatric surgeons even performed one successful case on a patient with a BMI of $82[18,19]$. Multiple retrospective studies emphasize the safety of robotic gynecologic surgery in morbidly obese women with no differences in perioperative outcomes with increasing BMI [20,21].

Robotic and conventional laparoscopic approaches have similar complication rates and length of hospital stay, however studies of both bariatric and benign gynecologic surgeries report that robotic-assisted surgery takes less operating time, particularly in super morbidly obese patients with a BMI greater than $50[3,22]$. Even without reducing task time, robot simulations demonstrate that surgeons experience less fatigue and mental stress with robotic versus conventional laparoscopic approaches [23].

Physical considerations for the surgeon pertain more to those operating on morbidly obese patients in which the thicker abdominal wall may limit the movement of trocars despite the greater force required to move the instruments. In contrast to conventional laparoscopy, robotic mechanical wrists may overcome these limitations by moving the torque of the instruments away from the abdominal wall [24]. Even in non-obese patients, conventional laparoscopy requires a great amount of manually dexterity to perform intracorporeal knot-tying due to loss of depth perception, tactile sense and visual obstruction, leading many surgeons to avoid conventional laparoscopic suturing in favor of suture devices [25,26]. Obesity adds to these challenges because only a small part of the instruments are external, leading to exaggerated internal movements when force is applied [27].

Technical challenges historically limited the use of conventional laparoscopic approaches in morbidly obese patients. Robotic platforms have made minimally invasive gynecologic surgery (MIGS) feasible for patients by increasing the rate of MIS in morbidly obese uterine cancer patients from $6 \%$ in $1993-2007$ to $57 \%$ in $2008-2012$ and $78 \%$ in the year 2012 alone with $69 \%$ of surgeries completed robotically [28]. MIGS has a slightly higher conversion rate to laparotomy with increasing BMI in laparoscopic procedures, however with robotic-assisted approaches, surgeons complete most these cases with MIS $[16,29]$. ACOG has clearly stated that regardless of BMI, surgeons should offer patients the least invasive procedure [17]. In order to provide MIS to obese, morbidly obese and super morbidly obese women, robotic surgery continues to gain increased interest among benign gynecologists.

\section{Preoperative Considerations}

\section{History and physical examination}

When performing a preoperative history and physical examination, topics that pertain more to the obese patient than the non-obese patient include: obstructive sleep apnea, smoking status, skin infections and estrogen therapy.

If a patient has known or suspected to have obstructive sleep apnea (OSA) or a difficult airway, consider a preoperative consultation with an anesthesiologist [17]. If she has a continuous positive airway pressure (CPAP) machine, she should bring it to the hospital on the day of surgery. She will need to maintain an open airway because obese women with and without OSA experience postoperative hypoxemia more frequently than non-obese women [30].

Current smoking also increases postoperative pulmonary complications by six-fold, so if present, encourage smoking cessation at least four weeks but ideally eight weeks prior to surgery [31]. 
Obese women may develop cutaneous bacterial or fungal infections underneath the breasts and pannus. Carefully inspect these areas and treat prior to surgery to reduce her risk of developing a SSI.

Since obese women have an increased risk of VTE, consider discontinuation of estrogen therapies. The American Association of Clinical Endocrinologists, The Obesity Society, and the American Society for Metabolic and Bariatric Surgery suggest that transdermal and oral estrogen therapy should be discontinued one cycle prior to surgery in premenopausal women and three weeks prior to surgery in postmenopausal women [32].

\section{Laboratory testing}

Laboratory testing prior to surgery should include a complete blood count and a basic metabolic panel. If the patient is diabetic, consider delaying the surgery if the hemoglobin A1c is greater than 7 percent or the fasting blood glucose concentration is greater than $110 \mathrm{mg} / \mathrm{dL}$ [32]. Obtain a cardiology clearance if the patient has any cardiac symptoms and a primary care physician clearance if she has any addition co-morbid medical conditions.

\section{Informed consent}

When obtaining informed consent, emphasize that the degree of obesity correlates with increased risk of surgical complications. Obese women have higher rates of SSI and overall infections $[9,10]$ Obese women have an increased risk of VTE and may require low molecular weight heparin or low-dose unfractionated heparin in addition to mechanical prophylaxis with intermittent pneumatic compression devices $[33,34]$. Obese women have an increased susceptibility to positional nerve injuries, including ulnar, sciatic, femoral, genitofemoral and brachial plexus nerve injuries that may result in unpredictable physical and psychological effects $[9,35]$ Obese women may not tolerate Trendelenburg positioning, which can decrease venous return, compromising pulmonary function and lead to cardiac arrhythmias $[29,36,37]$. Lastly, obese women have a higher risk of pre-peritoneal insufflation, which may make the surgery even more challenging and require repetitive or alternative attempts during surgery to gain abdominal access [15].

\section{On the Day of Surgery}

\section{Antibiotics}

Not only does obesity increase the risk of SSI, but increases in BMI may alter pharmacokinetics of antibiotic drugs 38 . The standard dose of cefazolin two grams needs to be increased to three grams if the patient weighs more than $120 \mathrm{~kg}[17,39]$.

The administration of an additional dose of antibiotic prophylaxis is needed if over four hours have elapsed from the preoperative dose or if blood loss exceeds $1500 \mathrm{~mL}$ [39-41].

ACOG recommends considering screening for and treatment of bacterial vaginosis (BV) five to seven days prior to hysterectomies, as BV can cause postoperative SSI40. With the high risk of SSI in obese women, some providers have suggested prophylactic treatment on the day of surgery with $500 \mathrm{mg}$ of IV metronidazole should be added to the routine antibiotics, even in women who do not test positive preoperatively $[42,43]$.

\section{Gastroesophageal reflux}

Extra care should be taken to prevent aspiration in obese patients because they have a lower $\mathrm{pH}$ (less than 2.5) and greater volume (more than $25 \mathrm{ml}$ ) of gastric juice compared to non-obese patients [44]. In addition to administering an acid-reducer, such as ranitidine $50 \mathrm{mg}$ IV 60 to $90 \mathrm{~min}$. prior to surgery, all obese patients regardless of port placement positioning should have an orogastric tube as an extra precaution [24].

\section{Anesthesia considerations}

The anesthesia team should be given time and space to properly care for the obese patient. They may need to place towels or ramps under the neck for intubation. They may also need to place the patient in the reverse Trendelenburg or beach chair position for intubation. The positioning should be tweaked until the external auditory meatus and the sternal notch are on the same level, this will facilitate the intubation.

A plan should be set in place, with the anesthesiologist, to address elevations of end tidal carbon dioxide (et $\mathrm{CO}_{2}$ ) levels during surgery. If et $\mathrm{CO}_{2}$ levels are between 25 and $33 \mathrm{~mm} \mathrm{Hg}$ while operating, then measures should be taken to decrease this level such asplacing the patient in reverse Trendelenburg, desufflation of the abdomen and administration of intravenous (IV) fluids [37].

Ensuring adequate IV access is essential, sometimes two different lines are needed, prior to the procedure.

Additionally, at the conclusion of the case, obese patients are usually extubated after being placed in a semi-recumbent position. They should be kept intubated if the anesthesiologist suspects laryngeal edema [24].

\section{Patient Positioning}

Patient positioning can make or break a case. As such, a gynecologist should take her or his time, and perform all the recommended steps. Obese patients may likely slide in the Trendelenburg position if appropriate precautions are not followed; this may place the patient and staff in serious danger if the robotic arms are docked. To avoid slipping, the surgeon should place an anti-slide material for the patient to lay on, such as a blue foam egg crate, gel or pad.

\section{Positioning of the legs}

When positioning the legs, the surgeon should use appropriately sized stirrups. Instead of the Yellowfin Allen stirrups, the use the Ultrafin Allen stirrups is advised as they are tailored for obese women. Additionally the surgeon should avoid candy cane stirrups and properly position the legs to avoid nerve injuries. Aligning the heal and knee with the contralateral shoulder is a good rule to follow to avoid nerve injuries.

\section{Tucking the arms}

When tucking the arms, the surgeon should consider cutting off the IV tubing clamps or wrapping them with gauze. Additionally, IV tubing and pulse oximeter cable away from the ulnar nerve region of the posterior elbow. The gown should be completely removed from the patient so that the fabric does not rest in the back or axillae. The arms should be wrapped in foam from the fingers to the elbow. The palms should be resting against the thighs. Consider using arm sleds, and place them as close to the stirrup brackets as possible to help support the hands. Verify with the anesthesia provider that the IV and blood pressure cuff are working correctly after both arms are wrapped and tucked to the sides using sleds. Use table width extenders if the shoulders upper arms or abdomen do not rest securely on the surgical bed. 


\section{Securing the chest}

Securing the chest adds an additional safety measure against the patient sliding up on the bed when in Trendelenburg, which may be very dangerous if the robotic arms are docked. To secure the chest, place a pillow-case wrapped foam strip above the nipples. Secure the foam and pillowcase from bedrail to bedrail with two layers of adhesive tape.

\section{Final touches}

Perform a "tilt test" to assess the positioning of the patient for slipping and tolerance by placing her in steep Trendelenburg. This is done prior to cleansing of the abdomen and draping the patient. The tilt test allows the surgeon to determine if additional adjustments to the patient position are needed to best protect her from slippage and improperly positioned legs.

The face of the patient will also need to be protected in robotic cases to avoid facial injuries. This can be done by using "face shields" commercially available, or by sliding a Mayo stand a few inches above the patient's nose, after the necessary degree of Trendelenburg has been determined.

Additionally, the surgeon has a responsibility to ensure that the sequential compression devices and Bair Hugger are functional and running prior to starting a surgery.

\section{Accessing the Abdomen}

Accessing the abdomen presents one of the most difficult steps in any laparoscopic surgery on the obese patient.

\section{Umbilical veress}

The Veress needle entry technique correlates with higher risks of preperitoneal insufflation and failed entry in the obese patient [45]. In women with a large pannus, abdominal anatomic margins are obscured in relation to the umbilicus, so entry should be supraumbilical. In women with no pannus, you may consider an infraumbilical entry. The umbilical stalk elevation (USE) technique reduces the rate of failed insufflations when establishing pneumoperitoneum in obese patients undergoing gynecologic laparoscopic procedures [46]. The USE involves isolating the umbilical stalk and elevating it with a towel clip with upward traction as the surgeon inserts the Veress needle at a 90 degree angle [46].

\section{Alternative techniques}

In morbidly obese women, alternative points of entry include transuterine or subcostal points, which both also significantly reduce insufflation failure rates [15].

Surgeons often choose Palmer's point because of less subcutaneous fat in the left upper quadrant insertion site than at the umbilicus. An optical trocar entry technique is an alternative to the use of a Veress needle at Palmer's point. Locate Palmer's point two to three $\mathrm{cm}$. below the subcostal margin in the midclavicular line. The surgeon should elevate the anterior wall with her or his non-dominant hand, then insert the optical trocar perpendicularly, carefully visualize each layer: subcutaneous fat, anterior rectus sheath, rectus muscle, posterior rectus sheath, preperitoneal fat and peritoneum.

A Cochrane review of 46 randomized controlled trials involving 7,389 patients found low quality evidence that direct trocar entry reduces failed entry and vascular injury over the Veress [45].

\section{Port placement}

After establishing pneumoperitoneum, the surgeon should select bariatric-sized $(16 \mathrm{~cm})$ robotic cannulas when placing the abdominal ports to avoid frequent dislodgement.

The surgeon should also "burp" the robotic ports. Burping means lift the ports away from the abdomen to tent the abdominal wall outward and create more space.

The surgeon should also use the bedside assistant and uterine manipulator to the maximal extents.

\section{Managing trendelenburg}

After positioning the patient, and before the abdomen is scrubbed, a tilt test should be performed. This will allow the surgeon to estimate if the patient will tolerate Trendelenburg during surgery. The tilt test should be repeated after a pneumoperitoneum is established and before the robotic arms are docked. Additionally, the surgeon should remember to only use the degree of Trendelenburg that is necessary to operate safely. Most of the time, this involves around fifteen to twenty degrees of Trendelenburg.

If the et $\mathrm{CO}_{2}$ level elevates in the middle of surgery, the abdomen should be desufflated, the robotic arms undocked, and the patient should be taken out of Trendelenburg while her et $\mathrm{CO}_{2}$ levels improve.

Additionally, a surgeon should consider operating with a lower $\mathrm{mmHg}$ to help with patient tolerance.

If a fan retractor is used to retract bowel, a surgeon may only need pressures between ten and twelve $\mathrm{mmHg}$ to obtain adequate visualization.

Finally, the surgeon should minimize the time a patient spends in Trendelenburg. For example, the patient should be taken out of Trendelenburg when closing the skin or performing a cystoscopy.

\section{Conclusions}

The advantages of MIS in the obese patient are well-documented. The robotic platform may further facilitate such surgeries with improved surgeon posture during surgery and overcome difficulties with dexterity and visualization that occur with conventional laparoscopy. To ensure success of robotic gynecologic surgery in the obese patient, a surgeon should follow the same routine every time. That routine should optimize the patient preoperatively, ensure proper and safe positioning, plan an abdominal entry technique that would minimize preperitoneal insufflation, use the proper surgical instrument use for the size of the patient and minimize the use of Trendelenburg.

\section{Author's Contributions}

The primary author contributed to literature review, first draft and final edits. The second author contributed to literature and second draft.

\section{Competing Interests}

The authors declare that they have no competing interests. 
Citation: Mikdachi H, SchreckA (2018) Robotic Surgery in the Obese Patient: Tips and Tricks for the Benign Gynecologist. Int J Gynecol Clin Pract 5: 146. https:// doi.org/10.15344/2394-4986/2018/146

\section{References}

1. Wright JD, Herzog TJ, Tsui J, Ananth CV Lewin SN, et al. (2013) Nationwide trends in the performance of inpatient hysterectomy in the United States. Obstet Gynecol 122: 233-241.

2. Orady M, Hrynewych A, Nawfal AK, Wegienka G (2012) Comparison of robotic-assisted hysterectomy to other minimally invasive approaches. JSLS 16: 542-548.

3. Billfeldt $\mathrm{NK}$, Borgfeldt $\mathrm{C}$, Lindkvist $\mathrm{H}$, Stjerndahl $\mathrm{JH}$, Ankardal $\mathrm{M}$, et al. (2018) A Swedish population-based evaluation of benign hysterectomy comparing minimally invasive and abdominal surgery. Eur J Obstet Gynecol Reprod Biol 222: 113-118.

4. Practice CoG (2017) Committee Opinion No 701: Choosing the Route of Hysterectomy for Benign Disease. Obstet Gynecol 129: e155-e159.

5. Aarts JW, Nieboer TE, Johnson N, Tavender E, Garry R, et al. (2015) Surgical approach to hysterectomy for benign gynaecological disease. Cochrane Database Syst Rev.

6. Lim PC, Crane JT, English EJ, Farnam RW, Garza DM, et al. (2016) Multicenter analysis comparing robotic, open, laparoscopic, and vaginal hysterectomies performed by high-volume surgeons for benign indications. Int J Gynaecol Obstet 133: 359-364.

7. Hales CM, Carroll MD, Fryar CD, Ogden CL (2017) Prevalence of Obesity Among Adults and Youth: United States, 2015-2016. NCHS Data Brief.

8. Organization WH (2018) Obesity and Overweight. Fact Sheet Web site.

9. Bamgbade OA, Rutter TW, Nafiu OO, Dorje P (2007) Postoperative complications in obese and nonobese patients. World J Surg 31: 556-560.

10. Suidan RS, He W, Sun CC, Zhao H, Fleming ND, et al. (2017) Impact of body mass index and operative approach on surgical morbidity and costs in women with endometrial carcinoma and hyperplasia. Gynecol Oncol 145 55-60.

11. Blikkendaal MD, Schepers EM, van Zwet EW, Twijnstra AR, Jansen FW et al. (2015) Hysterectomy in very obese and morbidly obese patients: a systematic review with cumulative analysis of comparative studies. Arch Gynecol Obstet 292: 723-738.

12. Colling KP, Glover JK, Statz CA, Geller MA, Beilman GJ, et al. (2015) Abdominal Hysterectomy: Reduced Risk of Surgical Site Infection Associated with Robotic and Laparoscopic Technique. Surg Infect 16: 498-503.

13. Mendivil AA, Rettenmaier MA, Abaid LN, Brown JV, Micha JP, et al. (2015) A comparison of open surgery, robotic-assisted surgery and conventional laparoscopic surgery in the treatment of morbidly obese endometria cancer patients. JSLS 19: e2014.00001.

14. Holtz G (1987) Laparoscopy in the massively obese female. Obstet Gynecol 69: 423-424

15. Pasic R, Levine RL, Wolf WM (1999) Laparoscopy in morbidly obese patients. J Am Assoc Gynecol Laparosc 6: 307-312.

16. Eltabbakh GH, Piver MS, Hempling RE, Recio FO (1999) Laparoscopic surgery in obese women. Obstet Gynecol 94: 704-708.

17. Practice CoG (2015) Committee opinion no. 619: Gynecologic surgery in the obese woman. Obstet Gynecol 125: 274-278.

18. Wilson EB, Sudan R (2013) The evolution of robotic bariatric surgery. World J Surg 37: 2756-2760.

19. Moser F, Horgan S (2004) Robotically assisted bariatric surgery. Am J Surg 188: 38S-44S.

20. Eddib A, Danakas A, Hughes S, Erk M, Michalik C, et al. (2014) Influence of Morbid Obesity on Surgical Outcomes in Robotic-Assisted Gynecologic Surgery. J Gynecol Surg 30: 81-86.

21. Gallo T, Kashani S, Patel DA, Elsahwi K, Silasi DA, et al. (2012) Roboticassisted laparoscopic hysterectomy: outcomes in obese and morbidly obese patients. JSLS 16: 421-427.

22. Edelson PK, Dumon KR, Sonnad SS, Shafi BM, Williams NN, et al. (2011) Robotic vs. conventional laparoscopic gastric banding: a comparison of 407 cases. Surg Endosc 25: 1402-1408.

23. Lee EC, Rafiq A, Merrell R, Ackerman R, Dennerlein JT, et al. (2005) Ergonomics and human factors in endoscopic surgery: a comparison of manual vs telerobotic simulation systems. Surg Endosc 19: 1064-1070.

24. Scheib SA, Tanner E, Green IC, Fader AN (2014) Laparoscopy in the morbidly obese: physiologic considerations and surgical techniques to optimize success. J Minim Invasive Gynecol 21: 182-195.

25. Croce $\mathrm{E}$, Olmi S (2000) Intracorporeal knot-tying and suturing techniques in laparoscopic surgery: technical details. JSLS 4: 17-22.
26. Pattaras JG, Smith GS, Landman J, Moore RG (2001) Comparison and analysis of laparoscopic intracorporeal suturing devices: preliminary results. J Endourol 15: 187-192.

27. Artuso D, Wayne M, Grossi R (2005) Use of robotics during laparoscopic gastric bypass for morbid obesity. JSLS 9: 266-268.

28. Leitao MM, Narain WR, Boccamazzo D, Sioulas V, Cassella D, et al. (2016) Impact of Robotic Platforms on Surgical Approach and Costs in the Management of Morbidly Obese Patients with Newly Diagnosed Uterine Cancer. Ann Surg Oncol 23: 2192-2198.

29. Cosin JA, Brett Sutherland MA, Westgate CT, Fang H (2016) Complications of Robotic Gynecologic Surgery in the Severely Morbidly Obese. Ann Surg Oncol 23: 4035-4041.

30. Ahmad S, Nagle A, McCarthy RJ, Fitzgerald PC, Sullivan JT, et al. (2008) Postoperative hypoxemia in morbidly obese patients with and without obstructive sleep apnea undergoing laparoscopic bariatric surgery. Anesth Analg 107: 138-143.

31. Bluman LG, Mosca L, Newman N, Simon DG (1998) Preoperative smoking habits and postoperative pulmonary complications. Chest 113: 883-889.

32. Mechanick JI, Kushner RF, Sugerman HJ, Gonzalez-Campoy JM, CollazoClavell ML, et al. (2008) American Association of Clinical Endocrinologists, The Obesity Society, and American Society for Metabolic \& Bariatric Surgery Medical guidelines for clinical practice for the perioperative nutritional, metabolic, and nonsurgical support of the bariatric surgery patient. Surg Obes Relat Dis 4: 109-184.

33. Miller K, Hell E (2003) Laparoscopic surgical concepts of morbid obesity. Langenbecks Arch Surg 388: 375-384.

34. Kearon C, Akl EA, Comerota AJ, Prandoni P, Bounameaux $\mathrm{H}$, et al. (2012) Antithrombotic therapy for VTE disease: Antithrombotic Therapy and Prevention of Thrombosis, 9th ed: American College of Chest Physicians Evidence-Based Clinical Practice Guidelines. Chest 141: e419S-e496S.

35. Berhane L, Kurman R, Smith S (1998) Lower extremity paralysis after operative laparoscopy from conversion disorder. A case report. J Reprod Med 43: 831-835.

36. Stephan JM, Goodheart MJ, McDonald M, Hansen J, Reyes HD, et al. (2015) Robotic surgery in super morbidly obese patients with endometrial cancer. Am J Obstet Gynecol 213: 49.e41-49.e48.

37. Srivastava A, Niranjan A (2010) Secrets of safe laparoscopic surgery: Anaesthetic and surgical considerations. J Minim Access Surg 6: 91-94.

38. Bratzler DW, Dellinger EP, Olsen KM, Perl TM, Auwaerter PG, et al. (2013) Clinical practice guidelines for antimicrobial prophylaxis in surgery. Am Health Syst Pharm 70: 195-283.

39. Bratzler DW, Dellinger EP, Olsen KM, Perl TM, Auwaerter PG, et al. (2013) Clinical practice guidelines for antimicrobial prophylaxis in surgery. Surg Infect 14: 73-156.

40. ACOG Practice Bulletin No. 195: Prevention of Infection After Gynecologic Procedures. Obstet Gynecol 131: e172-e189.

41. Swoboda SM, Merz C, Kostuik J, Trentler B, Lipsett PA, et al. (1996) Does intraoperative blood loss affect antibiotic serum and tissue concentrations? Arch Surg 131: 1165-1171.

42. McElligott KA, Havrilesky L, Myers ER (2011) Preoperative screening strategies for bacterial vaginosis prior to elective hysterectomy: a cost comparison study. Am J Obstet Gynecol 205: 500.e501-507.

43. Henriksson L, Colling-Saltin AS, Frick G, et al. Metronidazole prophylaxis to prevent infections after total abdominal hysterectomy. Acta Obstet Gynecol Scand 77: 116-119.

44. Vaughan RW, Bauer S, Wise L (1975) Volume and pH of gastric juice in obese patients. Anesthesiology 43: 686-689.

45. Ahmad G, Gent D, Henderson D, O'Flynn H, Phillips K, et al. (2015) Laparoscopic entry techniques. Cochrane Database Syst Rev 8: CD006583.

46. Ozdemir A, Gungorduk K, Ulker K, Yasar L, Ertas IE, et al. (2014) Umbilical stalk elevation technique for safer Veress needle insertion in obese patients: a case-control study. Eur J Obstet Gynecol Reprod Biol 180: 168-171. 